\title{
\begin{tabular}{l|l} 
Mibraries & DSpace@MIT
\end{tabular}
}

\author{
MIT Open Access Articles
}

\section{A thermal study of amorphous and textured carbon and carbon nitride thin films via transient grating spectroscopy}

The MIT Faculty has made this article openly available. Please share how this access benefits you. Your story matters.

Citation: Cornetto, Olivier et al. "A thermal study of amorphous and textured carbon and carbon nitride thin films via transient grating spectroscopy." Carbon 130 (April 2018): 355-361 (c) 2018 Elsevier Ltd

As Published: http://dx.doi.org/10.1016/j.carbon.2018.01.025

Publisher: Elsevier BV

Persistent URL: https://hdl.handle.net/1721.1/123893

Version: Original manuscript: author's manuscript prior to formal peer review

Terms of use: Creative Commons Attribution-NonCommercial-NoDerivs License 
Texturing $\mathrm{CN}$ and its thermal properties Abstract 


\section{Introduction}

The discovery of $\beta-C_{3} \mathrm{~N}_{4}$ by Liu and Cohen in the 90 s triggered a surge in interest in carbon nitride (CN) materials, as its computed hardness makes it harder than diamond $d^{1-2}$ with high thermal conductivity. ${ }^{3}$ Many attempted to obtain the $\beta$ phase of $\mathrm{CN}$ in a controllable way without much success, and attention turned towards the growth of amorphous CN (a-CN) thin films which exhibit improved hardness ${ }^{4}$ and elastic recovery ${ }^{5-6}$ when nitrogen is implanted into the carbon film. The growth of $\mathrm{CN}$ has been studied using various methods, such as $\mathrm{DCMS}^{6}$, $\mathrm{RF}^{\text {sputtering }}{ }^{7}$, ion plating ${ }^{8}$, pulsed laser deposition ${ }^{9}$, ion vapor deposition ${ }^{10}$, and ion beam assisted deposition ${ }^{11-12}$. Using the extensive amount of growth data on $\mathrm{CN}$, Hellgren et al. established a diagram defining the various microstructural formations of amorphous $\mathrm{CN}$ depending on nitrogen concentration and growth temperature ${ }^{13}$. They observed a fully amorphous region for any nitrogen concentration below temperatures of $200^{\circ} \mathrm{C}$, while a two-structure zone was observed at higher temperatures. This included graphitic structures for low nitrogen content (below 5 at. \%) to a fullerene-like structure for higher nitrogen content (up to 25 at. \%). In this study, we consider a novel way of growing amorphous $\mathrm{CN}$ films with various microstructures by using High Power Impulse Magnetron Sputtering (HiPIMS), using a carbon target reactively sputtered in nitrogen gas. HiPIMS is a relatively recent physical vapour deposition (PVD) method introduced by Kouznetsov et al. in $1999^{14}$. It can produce high power densities at the sputtered target surface, thus enhancing the deposition rate and the ionization rate of the plasma, facilitating bonding with nitrogen. Amorphous $\mathrm{CN}$ thin films have a unique nanostructural variation, in particular the fullerene-like structure which does not exist in pure carbon thin films. As such, it is expected that the microstructure would greatly affect the thermal properties of the material. However, no such work on the thermal properties of $\mathrm{CN}$ has been reported, and this study aims to provide some initial insights on the thermal behaviour of HiPIMS-grown CN. Various thin films were grown on silicon substrates using HiPIMS, such as amorphous carbon and carbon nitride as well as nanocrystalline carbon and $\mathrm{CN}$ with vertical ordering. The thermal diffusivity of each film was measured using transient grating spectroscopy $(\mathrm{TGS})^{\mathrm{A}}$ in order to compare the impact of the material's nanostructure on its thermal properties, as well as to benchmark the thermal performance of $\mathrm{CN}$ and assess the impact of nitrogen on its thermal performance.

\footnotetext{
A Maznev, A. A., Nelson, K. A., Rogers, J. A. Optical heterodyne detection of laser-induced gratings. Optics Lett., 23(16):1319 (1998).
} 


\section{Experimental setup}

\subsection{Sample preparation}

The samples were grown using HiPIMS applied to a 3" diameter carbon target of purity 99.995\%, except for the nanocrystalline graphite (NCG) sample which was obtained using the filtered cathodic vacuum arc (FCVA) method as described elsewhere ${ }^{15}$. For the amorphous carbon film, the carbon target was sputtered in argon gas, while for the $\mathrm{CN}$ samples, the sputtering occurred in a mixture of argon and nitrogen gases. Single crystal silicon (001) samples were loaded into the HiPIMS chamber and pumped down until the base pressure reached at least $10^{-6} \mathrm{mbar}$. Due to the introduction of argon and nitrogen to initiate the glow plasma discharge during growth, the pressure would increase to between $3 \cdot 10^{-3}$ to $7 \cdot 10^{-3} \mathrm{mbar}$. A Hipster 1 pulsed power supply from lonautics was used to generate the pulsed current waveform. Each sample was obtained using a different recipe, and the successful ones used for this study are described in Table 1 below:

\begin{tabular}{|c|c|c|c|c|c|c|c|c|c|}
\hline & $\begin{array}{l}\text { Sample } \\
\text { name }\end{array}$ & $\begin{array}{l}\text { Gas } \\
\text { mixture } \\
\text { (sccm) }\end{array}$ & $\begin{array}{l}\text { Growth } \\
\text { pressure } \\
\text { (mbar) }\end{array}$ & $\mathrm{T}\left({ }^{\circ} \mathrm{C}\right)$ & $\begin{array}{l}\text { Bias } \\
\text { voltage } \\
\text { (V) }\end{array}$ & $\begin{array}{l}\text { Pulse } \\
\text { current } \\
\text { (A) }\end{array}$ & $\begin{array}{l}\text { Pulse } \\
\text { frequency } \\
(\mathrm{Hz})\end{array}$ & $\begin{array}{l}\text { Pulse } \\
\text { voltage } \\
\text { (V) }\end{array}$ & $\begin{array}{l}\text { Peak } \\
\text { power } \\
\text { density } \\
\left(\mathrm{W} / \mathrm{cm}^{2}\right)\end{array}$ \\
\hline $\begin{array}{l}\text { Amorphous } \\
\text { carbon }\end{array}$ & $\mathrm{aC}$ & Ar:32 & $3.7 \times 10^{-3}$ & $25-80$ & 0 & 22 & 4000 & 750 & 180 \\
\hline $\begin{array}{l}\text { Nanocrystalline } \\
\text { graphite with } \\
\text { vertical } \\
\text { ordering }\end{array}$ & $\begin{array}{l}\text { NCG } \\
\text { (nano- } \\
\text { crystalline } \\
\text { graphite) }\end{array}$ & N/A & $\begin{array}{l}\text { Below } \\
1 \times 10^{-5}\end{array}$ & 600 & 200 & N/A & N/A & $N / A$ & N/A \\
\hline $\begin{array}{l}\text { Amorphous } \\
\text { carbon nitride }\end{array}$ & $\mathrm{aCN}$ & $\mathrm{N}: 40$ & $6.8 \times 10^{-3}$ & $25-45$ & 0 & 28 & 4000 & 650 & 122 \\
\hline $\begin{array}{l}\text { Nanocrystalline } \\
\text { graphitic } \\
\text { carbon nitride } \\
\text { with vertical } \\
\text { ordering }\end{array}$ & $\begin{array}{l}\text { flCN } \\
\text { (fullerene- } \\
\text { like CN }\end{array}$ & $\begin{array}{l}\text { Ar:32 } \\
N: 8\end{array}$ & $4.5 \times 10^{-3}$ & 350 & 500 & 1 & 4000 & 535 & 29 \\
\hline
\end{tabular}

Table 1 - Summary of the successful recipes used for the growth of carbon and carbon nitride thin films. All films were grown using HiPIMS, except the NCG sample which was grown using FCVA based on a technique reported elsewhere ${ }^{15}$.

The "room temperature growth" temperatures for amorphous $\mathrm{C}$ and amorphous $\mathrm{CN}$ actually ranged from $25-80^{\circ} \mathrm{C}$, which is due to plasma energy being dissipated as heat in the substrate holder and sample. The growth time was changed from one recipe to another in order to obtain approximately the same thickness of $150 \mathrm{~nm}$ for each sample, except for NCG which was grown using FCVA and is around $100 \mathrm{~nm}$ thick.

\subsection{Sample characterization}

Film microstructures were first analysed by a Witec Raman spectroscope, with a laser excitation wavelength of $532 \mathrm{~nm}$. The Raman spectra were acquired from $900 \mathrm{~cm}^{-1}$ to $1900 \mathrm{~cm}^{-1}$ and the data was analyzed using the software OriginPro 9 to do the necessary curve fitting based on the work by Ferrari et $\mathrm{al}^{16}$. regarding the Raman study of $\mathrm{CN}$ films. This study is an extension of the work he did on the threestage model in carbon thin films ${ }^{17}$. Using this model, one can resolve the microcrystalline structure of an amorphous carbon film using the Raman $G$ peak position, while the ratio between the $D$ and $G$ peaks yields information about the $\mathrm{sp}^{2} / \mathrm{sp}^{3}$ ratio and nanocrystal formation in the film. The entire signal was deconvoluted using two functions: the $G$ peak was fitted using a Breit-Wigner-Fano (BWF) function, while 
the $D$ peak was modelled with a Lorentzian function. The $D$ to $G$ peak ratio I(D)/I(G) was computed as the ratio between the maximum of the BWF and Lorentzian functions. As Raman cannot give information about atomic ordering, high-resolution transmission electron microscopy (HRTEM) and electron energy loss spectroscopy (EELS) were performed to resolve the atomic structure in the films as well as to confirm the $\mathrm{sp}^{2} / \mathrm{sp}^{3}$ ratio in each.

\subsection{Transient Grating Spectroscopy}

The effective surface thermal diffusivity of the films was measured using transient grating spectroscopy (TGS). This method utilizes a spatially periodic pulsed laser excitation to induce a "material excitation," comprised of a thermal-expansion-induced surface displacement as well as temperature-induced transient changes in reflectivity ${ }^{A, B}$. These excitations are detected by overlapping two beams of a continuous wave $(\mathrm{CW})$ probe laser onto the excited region, and monitoring the intensity in the first order diffraction of the probe. Optical heterodyne amplification is used to increase the strength of the recorded signal by spatially overlapping a reference oscillator with the diffraction beam path ${ }^{A, C}$. Signals recorded in this manner are comprised of components resulting from the thermal decay of both the reflectivity and surface displacement gratings, along with a superimposed surface acoustic wave (SAW) oscillating at a frequency depending on the material and surface layering structure ${ }^{D}$. The functional dynamics of the surface displacement and reflectivity decay differ, and the relative contribution of each component to the recorded signal can be controlled by the heterodyne phase between the diffracted signal and reference oscillator ${ }^{\mathrm{E}}$. However, the rate of this decay is uniquely determined by the thermal diffusivity of the sample in question and the imposed excitation wavelength, such that decay profiles of this type may be fit to extract values for the effective thermal diffusivity of bulk or layered systems.

Here, a 300 ps pulse length, $532 \mathrm{~nm}$ laser with an excitation spot size of $225 \mu \mathrm{m}$, and an imposed grating wavelength of $7.41 \mu \mathrm{m}$ was rastered across the film surface to obtain spatially averaged measurements of each film. Probing the transient grating was accomplished using a $785 \mathrm{~nm}$, quasi-CW laser modulated to a duty cycle of $20 \%$ at the pump laser repetition rate $(1 \mathrm{kHz})$ to reduce specimen heating, and a spot size of $175 \mu \mathrm{m}$ placed at the centre of the excited spot. Samples were kept in rough vacuum (<15 mTorr) to simplify the measurements by removing any effects of excited acoustic waves in air and measured at ambient room temperature. More information about the TGS setup used for these experiments can be found in the work of Dennett and Short ${ }^{\mathrm{c}}$. The background noise during measurement was minimized by measuring each sample ten times and averaging the results. Once the decay curves were obtained, they were processed by curve fitting in order to compute the thermal diffusivity.

\footnotetext{
B J. A. Johnson and A. A. Maznev and M. T. Bulsara and E. A. Fitzgerald and T. C. Harman and S. Calawa and C. J. Vineis and G. Turner and K. A. Nelson. Phase-controlled, heterodyne laser-induced transient grating measurements of thermal transport properties in opaque material. J. Appl. Phys., 111(2) (2012).

C Dennett, C., Short, M. P. "Time-resolved, dual heterodyne phase collection transient grating spectroscopy." Appl. Phys. Lett. 110:211106 (2017).

D J. A. Rogers and A. A. Maznev and M. J. Banet and K. A. Nelson. Optical Generation and Characterization of Acoustic Waves in Thin Films: Fundamentals and Applications. Annu. Rev. Mater. Sci., 30(1):117 (2000).

E J. A. Johnson and J. K. Eliason and A. A. Maznev and T. Luo and K. A. Nelson. Non-diffusive thermal transport in GaAs at micron length scales. J. Appl. Phys., 118(15):155104 (2015).
} 


\section{Results and discussion}

\subsection{Microstructure analysis}

Raman spectroscopy was performed on each material, in order to confirm that the film structure is indeed observed throughout the whole sample. Figure 1 shows the Raman response of the NCG sample and its curve fitting computation for the $D$ and $G$ peaks. In the case of NCG, the I(D)/I(G) ratio is 1.28 , with a $G$ peak centred at $1585 \mathrm{~cm}^{-1}$. Associating this data with the three-stage model devised by Ferrari and Robertson ${ }^{17}$, the NCG microstructure can be deduced fairly accurately. In this case, the NCG sample has a structure in between stages 1 and 2, which is the characteristic response for a nanocrystalline graphite film with $100 \% \mathrm{sp}^{2}$ content, and is in agreement with the TEM image seen in Figure 3a. However, Raman analysis of the film cannot provide any information on the orientation of the nanocrystals, only TEM can measure this.

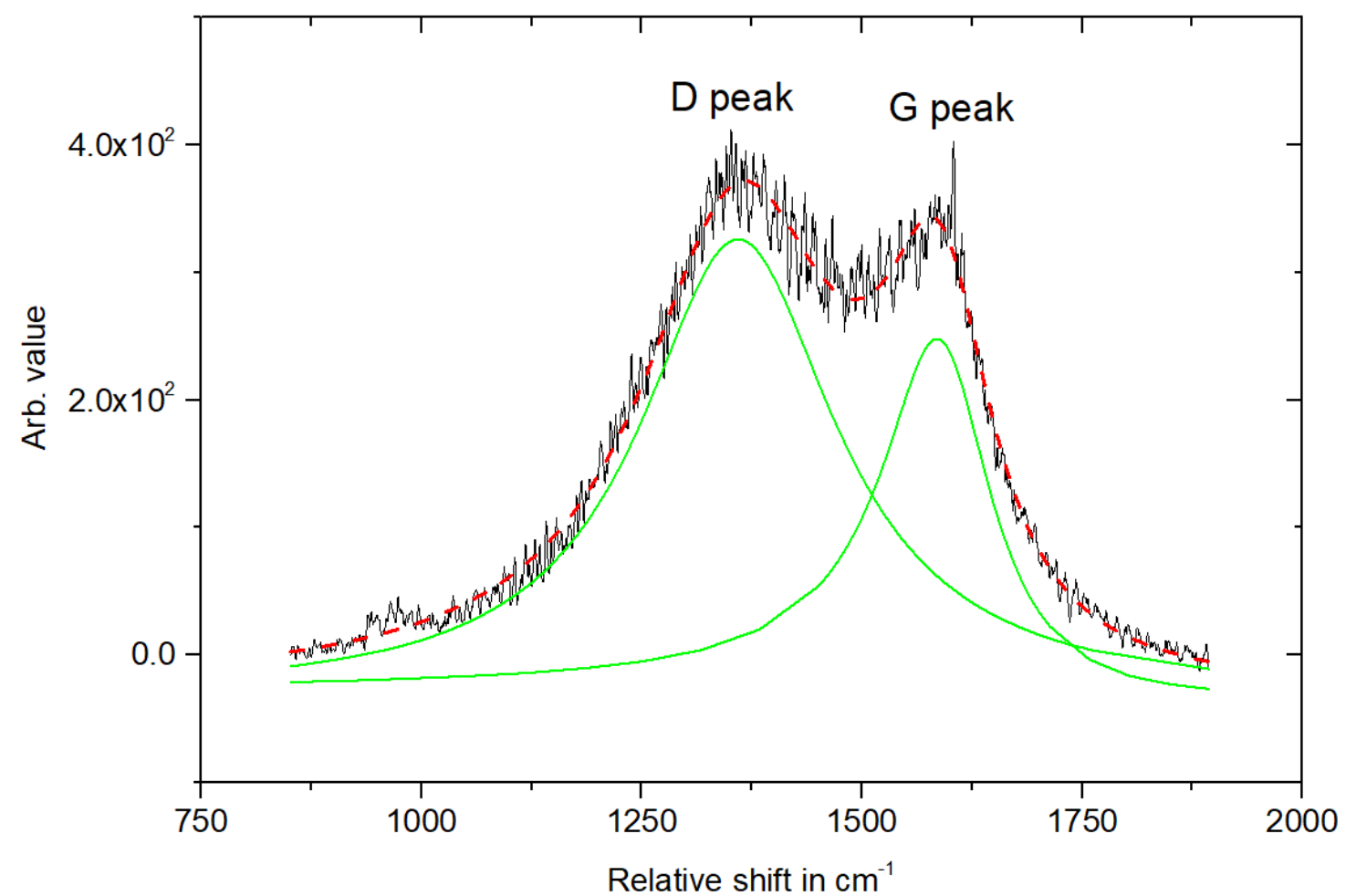

Figure 1: Raman spectrum of the NCG sample (noisy line) and its fit curve in thick green colour (Lorentzian for the D peak and BWF for the $\mathrm{G}$ peak). The red dotted line is the sum of both the Lorentzian and BWF fit, which is a close match to the actual Raman response. $I(D) / I(G)$ is 1.28 and the $G$ peak centre is located at $1585 \mathrm{~cm}^{-1}$

The other three materials were analyzed with Raman In a similar fashion to NCG, and their resulting responses are shown together with that of NCG in Figure 2. The microstructure for each material was determined using the $I(D) / I(G)$ ratio and the $G$ peak position. For amorphous carbon, the $I(D) / I(G)$ ratio is 0.67 and the $\mathrm{G}$ peak position is $1565 \mathrm{~cm}^{-1}$, indicating an amorphous structure with high $\mathrm{sp}^{2}$ content (approximately 90\%). In the case of the $\mathrm{CN}$ films, the three-stage model is slightly different as the nitrogen in the film will change the vibrational patterns, and Ferrari et al. modified their model to account for that ${ }^{16}$. Using this updated model, fICN and aCN Raman spectra were interpreted to have peak ratios of 1.17 and 0.97 respectively, while their $\mathrm{G}$ peak locations wre $1564 \mathrm{~cm}^{-1}$ and $1570 \mathrm{~cm}^{-1}$. For aCN, this implies a fully amorphous film with high $\mathrm{sp}^{2}$ content (approximately 90\%), while the higher peak ratio for fICN indicates an amorphous film with graphitic nanocrystals and the same amount of $\mathrm{sp}^{2}$ content. The results 
are in agreement with the TEM images in Figure 3, but as for NCG, neither the crystalline orientation nor the nitrogen content can be obtained with Raman spectroscopy. TEM/EELS is required to determine this information.

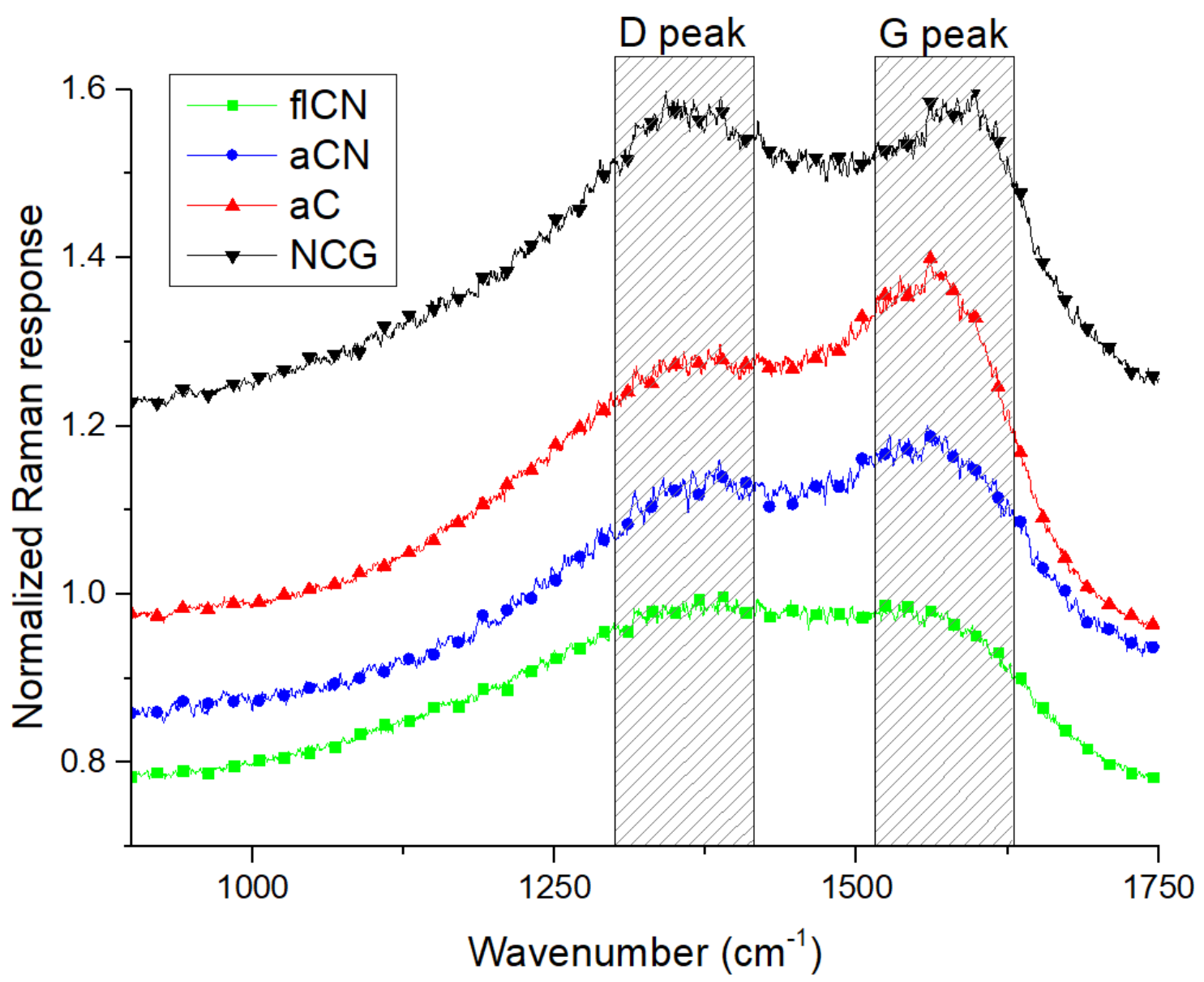

Figure 2: Raman spectra for all four materials in this study. The $D$ and $G$ peak regions are shown in here. Each spectrum shows a different shift for the G peak, which gives insights into the nanocrystalline structure of each film.

Cross sectional TEM was performed on all materials to confirm the data observed with Raman. HRTEM images for both the $\mathrm{aC}$ and the $\mathrm{aCN}$ films is shown in Figure 3. Both samples exhibit fully amorphous structures without any visible atomic arrangement. The EELS measurements provided the $\mathrm{sp}^{2}$ content in the films, with a value of $81 \%$ for the aC sample, and $93 \%$ for the aCN sample. In addition, EELS revealed a nitrogen content of 14.79 at. \% in the aCN film. 


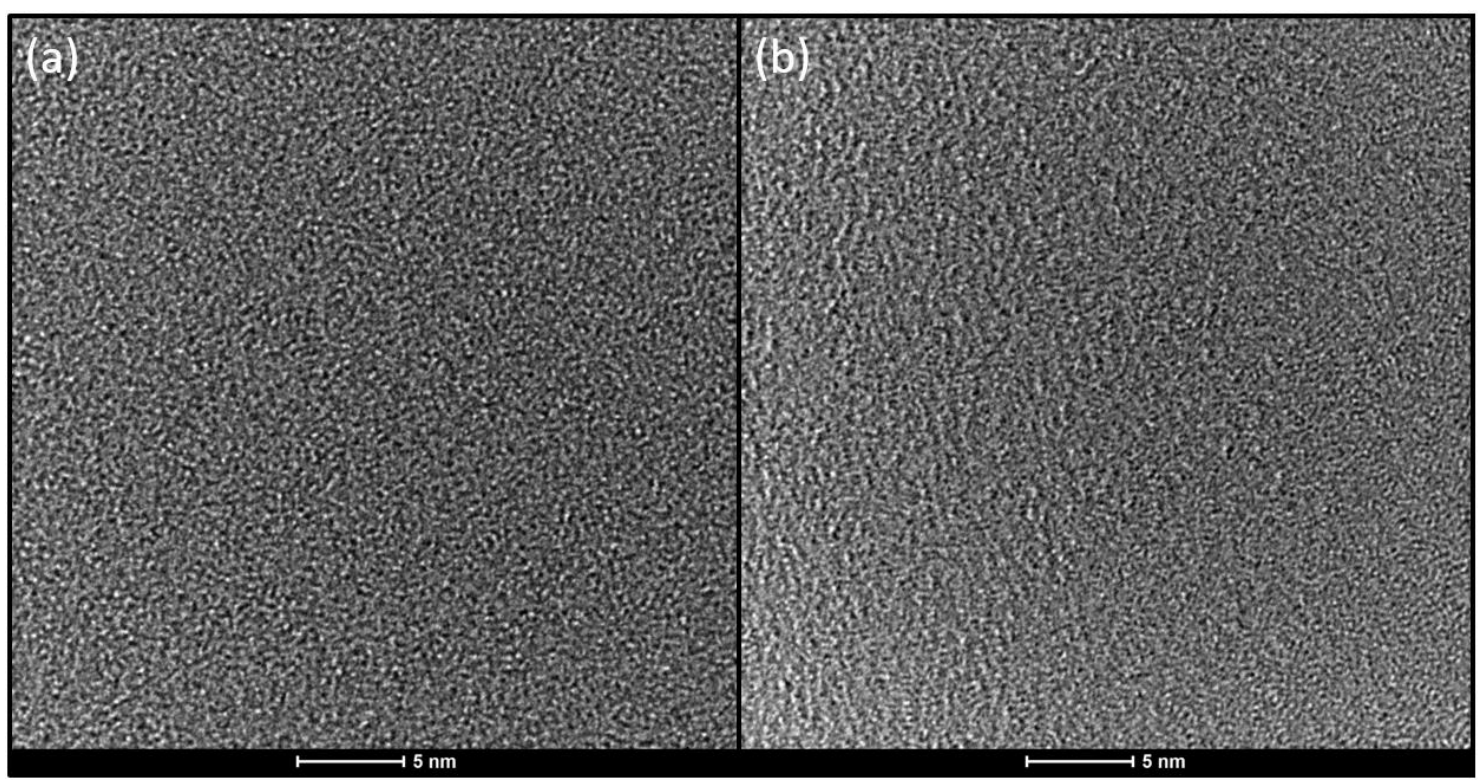

Figure 3: (a) FIBTEM of an amorphous carbon film. EELS measurements measured a $81 \% \mathrm{sp}^{2}$ content in the film. (b) FIBTEM of an amorphous carbon nitride film. EELS measurements reveal a $93 \% \mathrm{sp}^{2}$ content in the film.

Similarly to $\mathrm{aC}$ and aCN, NCG and fICN were observed with TEM with results in Figure 4. In contrast with $\mathrm{aC}$ and $\mathrm{aCN}$, the films here contain some form of ordering, with the inset showing the computed FFT for both images. Figure $4 a$ is a picture of the NCG sample, and vertically oriented graphitic planes can clearly be identified. The FFT confirms the orientation by the two partial circles indicating a periodic arrangement in the planar direction with a spacing of $0.34 \mathrm{~nm}$ per period, which corresponds to the inter-planar distance between two (002) graphitic planes. In Figure 4b, a TEM image of the fICN is shown and some ordering can be observed but to a lower degree compared to the NCG film in Figure 4a. The fullerene-like structure is induced by the additional nitrogen atoms disrupting the graphitic planes. Unlike a pure graphitic film made of carbon atoms in a hexagonal pattern, the nitrogen atoms replace some of the carbon atoms, increasing the $\mathrm{sp}^{3}$ content in the film and sometimes changing the local atomic structure from hexagonal to pentagonal as a structural defect ${ }^{4,20}$. This change would induce buckling in the basal planes, inducing circular shapes as seen in the TEM image in Figure $4 \mathrm{~b}$. The FFT in the inset of Figure $4 \mathrm{~b}$ shows a circular ring with a distance cycle of $0.44 \mathrm{~nm}$. The ring indicates random orientation, while the distance of $0.44 \mathrm{~nm}$ seems higher than the expected (002) inter-planar distance of $0.33 \mathrm{~nm}$. This is attributed to the graphitic plane buckling as described above, preventing a proper stacking with minimal distances. EELS shows a $82 \% \mathrm{sp}^{2}$ content for fICN, as well as a nitrogen content of 22.18 at. \% in the film. 


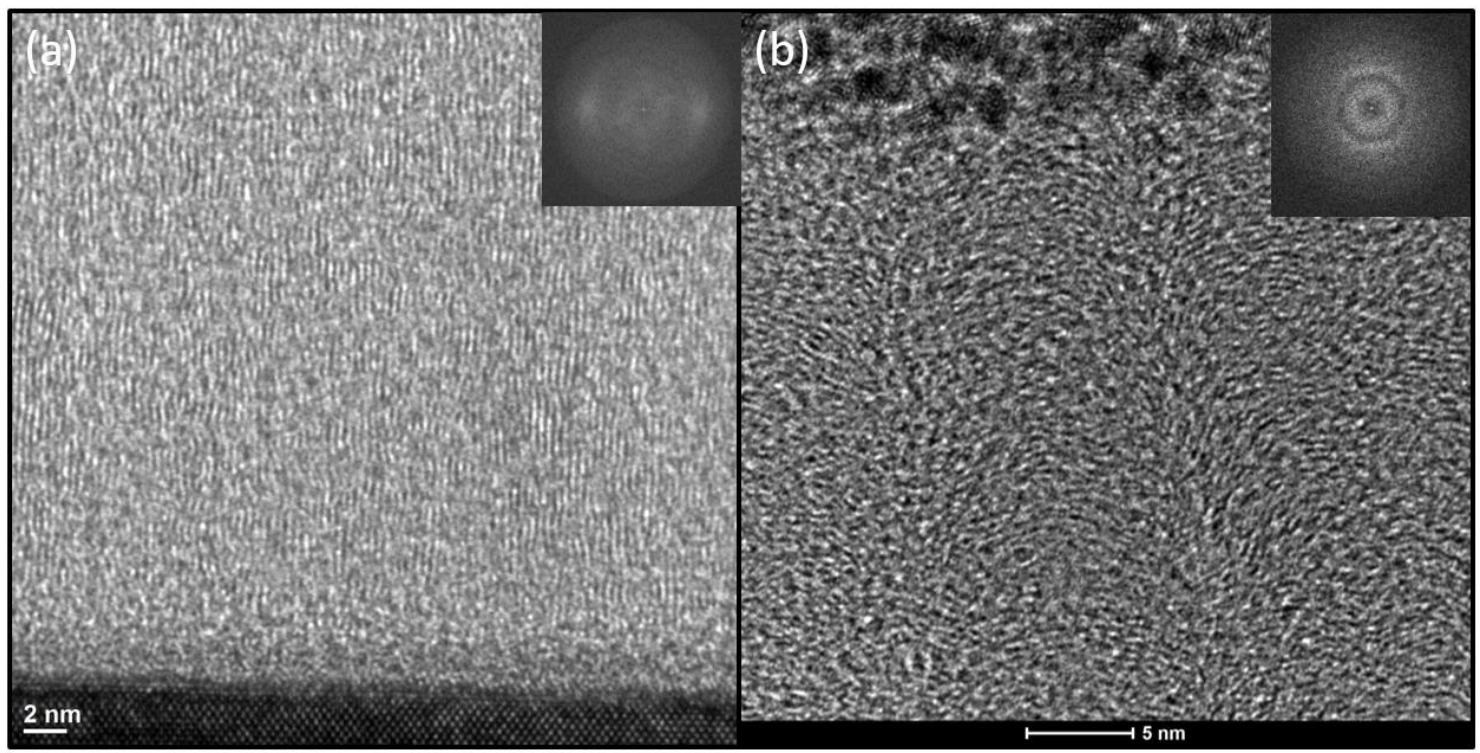

Figure 4: (a) FIBTEM of the NCG film with a $100 \% \mathrm{sp}^{2}$ content. The computed FFT image in the inset shows partial circles indicating an inter-planar distance of $0.34 \mathrm{~nm}$ corresponding to the [002] direction. (b) FIBTEM of the fullerene-like carbon nitride film. EELS measurements give a $88 \% \mathrm{sp}^{2}$ content in the film, with the inset showing the computed FFT of the image and its circle indicating an inter-planar distance of $0.44 \mathrm{~nm}$. In the end, the conclusions drawn from both the TEM images and the Raman responses paint a consistent picture of the microcrystalline structure of the four films.

\subsection{Thermal diffusivity characterization}

After characterizing the samples' microstructures, TGS was used to measure their effective thermal diffusivities. Figure 5 shows characteristic signals retrieved from each film when excited by a transient thermal grating. The complete form for the heterodyne amplified response, $I(t, \phi)$, is given by Johnson et al. as ${ }^{\mathrm{B}}$ :

$$
I(t, \phi)=A\left[r^{\prime}(t) \cos \phi-\left(r^{\prime \prime}(t)-2 k_{p} u(t) \cos \beta_{p}\right) \sin \phi\right],
$$

where $A$ is a laser-power-dependent amplitude constant, $r(t)=r_{0}\left[1+r^{\prime}(t)+i r^{\prime \prime}(t)\right]$ is the complex reflectivity, $\phi$ is the heterodyne phase, $k_{p}$ is the optical wavevector of the probe laser, $u(t)$ is the surface displacement, and $\beta_{p}$ is the angle of incidence of the probe beam onto the sample. By solving the heat diffusion equation and the equation of thermo-elasticity for a periodic surface excitation, Kading et al. show that the reflectivity and displacement dynamics take the following forms ${ }^{\mathrm{F}}$ :

$$
\begin{aligned}
& \left\{r^{\prime}(t), r^{\prime \prime}(t)\right\} \propto \frac{1}{\sqrt{t}} e^{-q^{2} \alpha t} \\
& u(t) \propto \operatorname{erfc}(q \sqrt{\alpha t})
\end{aligned}
$$

where $q=2 \pi / \Lambda$ and $\Lambda$ is the grating spacing, $\alpha$ is the isotropic thermal diffusivity, and $\operatorname{erfc}(x)$ is the complimentary error function. The form of Eq. (3) only accounts for the thermal decay of the surface displacement and does not include a component for the imposed SAW oscillation, which is also present in $u(t)$.

\footnotetext{
F Kading, O.W. and H. Skurk and A.A. Maznev and E. Matthias. Transient thermal gratings at surfaces for thermal characterization of bulk materials and thin films. Appl. Phys. A, 61(3):253 (1995).
} 
Each measurement is collected by using the method described in the work of Dennett et al. ${ }^{\mathrm{C}}$, setting the heterodyne phases to $\phi=\left\{\frac{\pi}{2},-\frac{\pi}{2}\right\}$. Signals collected in this way will include contributions to the thermal decay from both the reflectivity and displacement components of the response, and can be fit to the following form:

$$
I_{S}(t)=A\left[\operatorname{erfc}(q \sqrt{\alpha t})-\frac{\beta}{\sqrt{t}} e^{-q^{2} \alpha t}\right]
$$

where $\beta$ describes the relative amplitude of the displacement and reflectivity contributions to the response with a known value of $q$ to determine the effective thermal diffusivity of a film-substrate system. The thermal penetration depth of the excitation is $\Lambda / \pi$, such that the measured diffusivity will contain contributions from both the $\mathrm{C} / \mathrm{CN}$ film and the Si substrate ${ }^{\mathrm{F}}$. At the short excitation wavelengths used here, a suppression in the measured value for thermal diffusivity is to be expected due to the exclusion of long-wavelength ballistic phonons from the thermal relaxation process ${ }^{\mathrm{E}}$. This results in a measured value for a pure Si substrate reduced by about 30\% compared to its bulk value. Figure 5 shows representative TGS measurements of each of the four film-substrate systems measured.
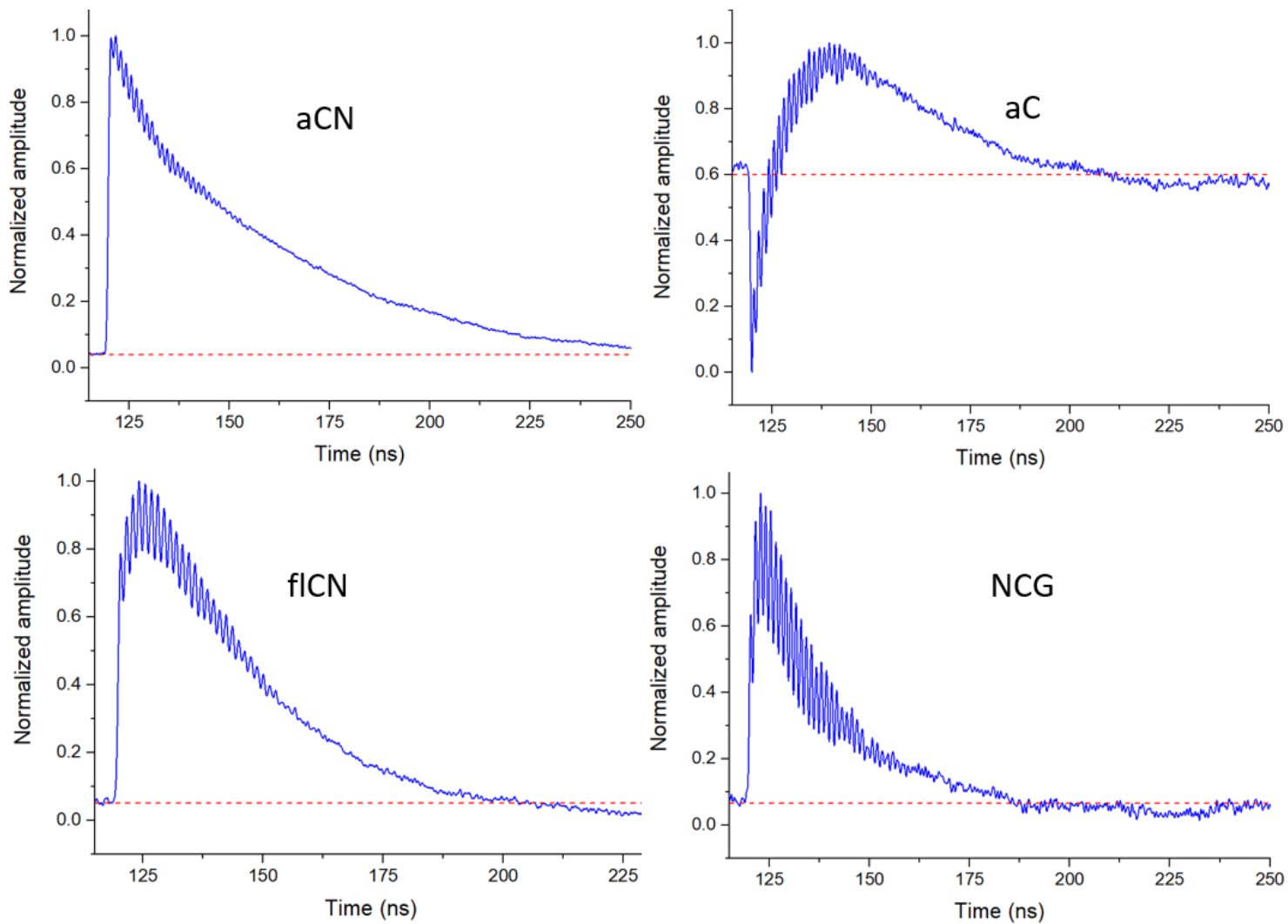

Figure 5: Surface acoustic waves decay after excitation by the projected pump laser grating. The exponential decay curve is input into a fitting model in order to compute the thermal diffusivity of the film-substrate system. The small sinusoidal oscillations are due to the elastic properties of the material. The dashed line in each plot is the zero line. 


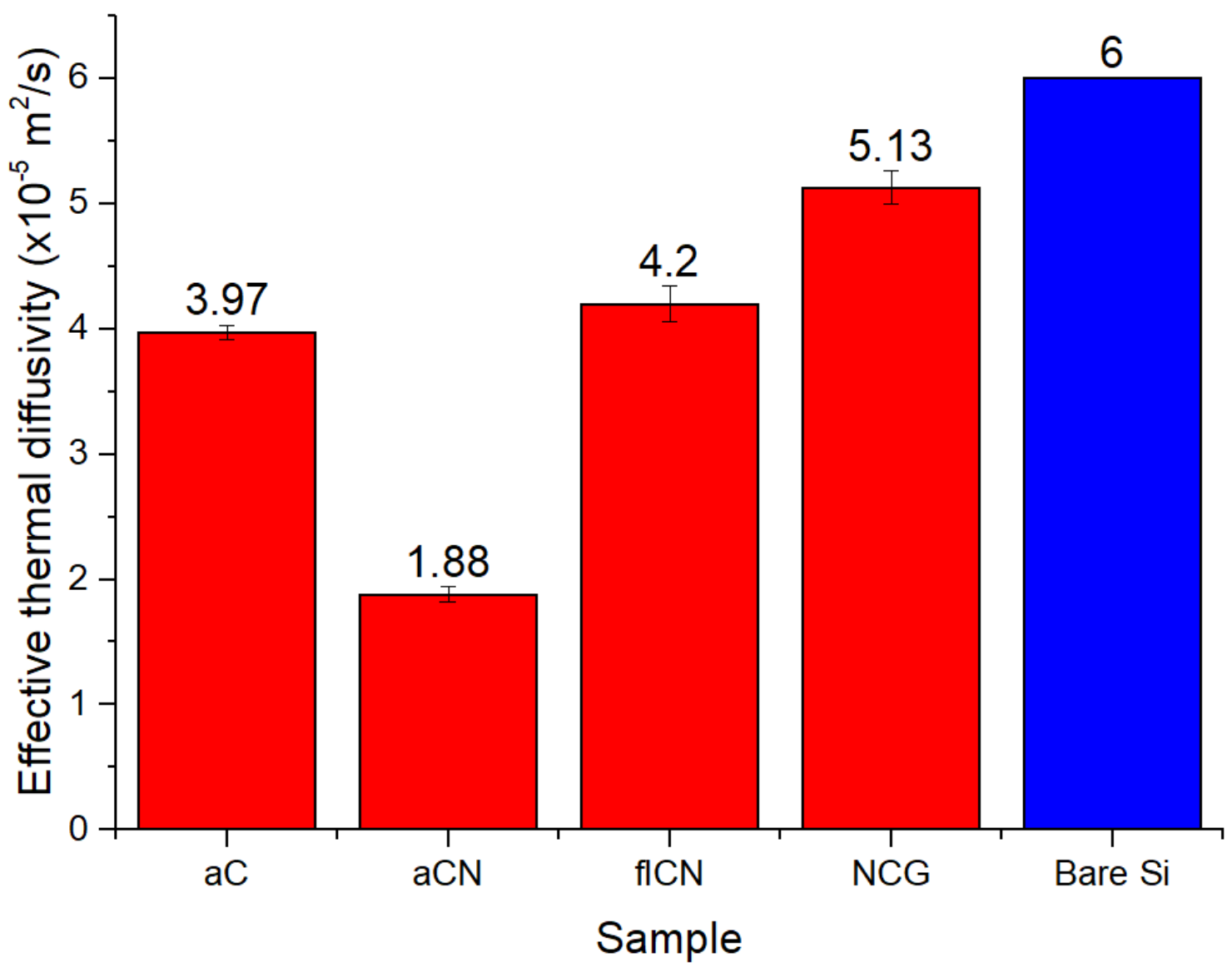

Figure 6: Thermal diffusivity value for each sample. As the grating wavelength is $7.4 \mu \mathrm{m}$ deep, the thermal diffusivity value is made of a two-layer system made of the $150 \mathrm{~nm}$ thick $\mathrm{C}$ or $\mathrm{CN}$ film with several microns of $\{001\}$ silicon, which has a thermal diffusivity of around $6 \times 10^{-5} \mathrm{~m}^{2} / \mathrm{s}$.

The best-fit thermal diffusivity numbers shown in Figure 6 are for the whole thin film-substrate system, and it can be seen that they all exhibit a lower thermal diffusivity compared to the bare Si substrate. Considering that the laser excitation wavelength is $7.41 \mu \mathrm{m}$, most of the heating energy will be contributed to the $\mathrm{Si}$ substrate, which implies that most of the thermal diffusivity contribution comes from the substrate ${ }^{G}$. Based on the work of Seremus et al., the thermal diffusivities of the roughly $150 \mathrm{~nm}$ thick films are roughly a factor of three lower than the directly measured film-substrate values. For the amorphous films, it can be seen that introducing around 15 at. \% nitrogen halves the absolute thermal diffusivity compared to a pure amorphous carbon film. This decrease is most likely due to the increased disorder in the film nanostructure due to the nitrogen incorporation. As is to be expected, the vertically ordered NCG sample has a higher thermal diffusivity than amorphous carbon as observed in a previous study. ${ }^{22}$ This improvement is due to the well-ordered fully $\mathrm{sp}^{2}$ hybridized basal planes, facilitating phonon transport within the film. By contrast, the aC films have no clear phonon propagation path, inducing much phonon scattering. When it comes to the carbon nitride samples, the same conclusion cannot be reached as they both have a similar nanostructure hybridization with around $90 \% \mathrm{sp}^{2}$ bonding. The only difference

\footnotetext{
${ }^{G} \mathrm{~J}$. Sermeus and B. Verstraeten and R. Salenbien and P. Pobedinskas and K. Haenen and C. Glorieux. Determination of elastic and thermal properties of a thin nanocrystalline diamond coating using all-optical methods. Thin Solid Films, 590:284 (2015).
} 
lies in the crystalline formation, where fICN would contain some patterns of structural organization with some pseudo-aligned graphitic planes as observed in the TEM images.

Both films containing crystalline ordering performed better thermally than their amorphous counterparts, suggesting the crystalline order is a main driver for improved thermal diffusivity via improved phonon transport. Also, TGS only computes the thermal diffusivity in the in-plane direction here, and as amorphous films are intrinsically anisotropic, this is not necessarily true for the NCG and fICN films. In these more ordered films some anisotropy is to be expected, as seen in another study with vertically ordered boron nitride thin films ${ }^{23}$. In addition to the phonon transport, thermal transport can also occur through the electrons, which can be quite dominant in electrically conductive materials such as metals where the empirical Wiedemann-Franz law ${ }^{24}$ states the electrical and thermal conductivities are linearly related at constant temperature. The sheet resistances of all four films were computed in order to find a link between the thermal diffusivity and the sheet resistivity. Both amorphous samples were found to be quite resistive, with a sheet resistances of $10^{6} \mathrm{Ohm} / \mathrm{cm}$ for aC and $10^{2} \mathrm{Ohm} / \mathrm{cm}$ for aCN, while fICN exhibited around $10^{-2} \mathrm{Ohm} / \mathrm{cm}$ and NCG was at around $1 \mathrm{Ohm} / \mathrm{cm}$. Overall, the amorphous samples which have electrical sheet resistances several orders of magnitude higher than the other samples were found to indeed have lower thermal diffusivities. Yet, when comparing both amorphous samples directly, the sheet resistance of $\mathrm{aC}$ is still be 4 orders or magnitude higher than aCN even though its thermal diffusivity is twice as high. This lack of correlation between thermal diffusivity and electrical conductivity points to phonon-dominated heat transport in those materials, where the introduction of nitrogen in the material greatly disrupts the nanostructure of the film and plays a dominant role in reducing the thermal diffusivity. 


\section{Conclusion}

A new way to grow carbon nitride using HiPIMS was used to texture the material from amorphous to fullerene-like in atomic structure. TGS was used to obtain in-plane thermal diffusivities, and to compare them with carbon samples with similar crystalline structures. As expected, amorphous films exhibited poorer thermal diffusivity than the nanocrystalline ones, due to phonon transport hindrances in amorphous samples. In addition, amorphous carbon nitride showed an even lower thermal diffusivity than amorphous carbon. The effect of heat transport via electrons to explain this observation was disproved, as aC was four orders of magnitude more electrically resistive than aCN while still having better thermal performance. Hence the lower thermal diffusivity in amorphous carbon nitride could only be attributed to phonon transport being disrupted by the nitrogen atoms increasing disorder in the atomic structure. Finally this study is the first work performed on the thermal properties of carbon nitride thin films, and it demonstrated that nitrogen doping plays a crucial role in the thermal properties of carbon nitride thin films to help engineer the thermal properties of this material. 


\section{References}

1. Sung, C.-M.; Sung, M., Carbon nitride and other speculative superhard materials. Mater. Chem. Phys. 1996, $43(1), 1-18$.

2. Liu, A. Y.; Cohen, M. L., Structural properties and electronic structure of low-compressibility materials: $\beta$-Si3N4 and hypothetical $\beta$-C3N4. Physical Review B 1990, 41 (15), 10727-10734.

3. Morelli, D. T.; Heremans, J. P., Thermal conductivity of germanium, silicon, and carbon nitrides. Appl. Phys. Lett. 2002, 81 (27), 5126-5128.

4. Sjöström, H.; Stafström, S.; Boman, M.; Sundgren, J. E., Superhard and Elastic Carbon Nitride Thin Films Having Fullerenelike Microstructure. Phys. Rev. Lett. 1995, 75 (7), 1336-1339.

5. Li, D.; Chung, Y. W.; Wong, M. S.; Sproul, W. D., Nano- indentation studies of ultrahigh strength carbon nitride thin films. J. Appl. Phys. 1993, 74 (1), 219-223.

6. Broitman, E.; Hellgren, N.; Wänstrand, O.; Johansson, M. P.; Berlind, T.; Sjöström, H.; Sundgren, J. E.; Larsson, M.; Hultman, L., Mechanical and tribological properties of $\mathrm{CNx}$ films deposited by reactive magnetron sputtering. Wear 2001, $248(1-2), 55-64$.

7. Cuomo, J. J.; Leary, P. A.; Yu, D.; Reuter, W.; Frisch, M., Reactive sputtering of carbon and carbide targets in nitrogen. Journal of Vacuum Science \& Technology 1979, 16 (2), 299-302.

8. Taki, Y.; Kitagawa, T.; Takai, O., Shielded arc ion plating and structural characterization of amorphous carbon nitride thin films. Thin Solid Films 1997, 304 (1-2), 183-190.

9. Yap, Y. K.; Kida, S.; Aoyama, T.; Mori, Y.; Sasaki, T., Influence of negative dc bias voltage on structural transformation of carbon nitride at $600^{\circ} \mathrm{C}$. Appl. Phys. Lett. 1998, 73 (7), 915-917.

10. Fujimoto, F.; Ogata, K., Formation of Carbon Nitride Films by Means of lon Assisted Dynamic Mixing (IVD) Method. Japanese Journal of Applied Physics 1993, 32 (Part 2, No. 3B), L420-L423.

11. Kohzaki, M.; Matsumuro, A.; Hayashi, T.; Muramatsu, M.; Yamaguchi, K., Preparation of carbon nitride thin films by ion beam assisted deposition and their mechanical properties. Thin Solid Films 1997, 308-309 (0), 239-244.

12. Bousetta, A.; Lu, M.; Bensaoula, A.; Schultz, A., Formation of carbon nitride films on Si(100) substrates by electron cyclotron resonance plasma assisted vapor deposition. Appl. Phys. Lett. 1994, 65 (6), 696-698.

13. Hellgren, N.; Johansson, M. P.; Broitman, E.; Hultman, L.; Sundgren, J.-E., Role of nitrogen in the formation of hard and elastic $<$ span class="aps-inline-formula" $><$ math $><$ mrow $><$ msub $><$ mrow $><$ mi mathvariant="normal" $>\mathrm{CN}</$ mi $><$ /mrow $><$ mrow $><$ mi $>\mathrm{x}</$ mi $><$ /mrow $><$ /msub $><$ /mrow $>/$ math $><$ span $>$ thin films by reactive magnetron sputtering. Physical Review $B$ 1999, 59 (7), 5162-5169.

14. Kouznetsov, V.; Macák, K.; Schneider, J. M.; Helmersson, U.; Petrov, I., A novel pulsed magnetron sputter technique utilizing very high target power densities. Surf. Coat. Technol. 1999, 122 (2-3), 290-293.

15. Teo, E. H. T.; Kulik, J.; Kauffmann, Y.; Kalish, R.; Lifshitz, Y., Nanostructured carbon films with oriented graphitic planes. Appl. Phys. Lett. 2011, 98 (12).

16. Ferrari, A. C.; Rodil, S. E.; Robertson, J., Interpretation of infrared and Raman spectra of amorphous carbon nitrides. Physical Review B 2003, 67 (15), 155306.

17. Ferrari, A. C.; Robertson, J., Interpretation of Raman spectra of disordered and amorphous carbon. Physical Review B 2000, 61 (20), 14095-14107.

18. Maznev, A. A.; Nelson, K. A.; Rogers, J. A., Optical heterodyne detection of laser-induced gratings. Opt. Lett. 1998, $23(16), 1319-1321$.

19. Dennett, C. A.; Short, M. P., Time-resolved, dual heterodyne phase collection transient grating spectroscopy. Appl. Phys. Lett. 2017, 110 (21), 211106.

20. Hultman, L.; Neidhardt, J.; Hellgren, N.; Sjöström, H.; Sundgren, J.-E., Fullerene-like Carbon Nitride: A Resilient Coating Material. MRS Bull. 2003, 28 (03), 194-202.

21. Kading, O. W.; Skurk, H.; Goodson, K. E., Thermal conduction in metallized silicon-dioxide layers on silicon. Appl. Phys. Lett. 1994, 65 (13), 1629-1631. 
22. Shakerzadeh, M.; Samani, M. K.; Khosravian, N.; Teo, E. H. T.; Bosman, M.; Tay, B. K., Thermal Conductivity of Nanocrystalline Carbon Films Studied by Pulsed Photothermal Reflectance. Carbon 2012, 50 (3), 1428-1431.

23. Cometto, O.; Samani, M. K.; Liu, B.; Sun, S.; Tsang, S. H.; Liu, J.; Zhou, K.; Teo, E. H., Control of Nanoplane Orientation in voBN for High Thermal Anisotropy in a Dielectric Thin Film: A New Solution For Thermal Hotspot Mitigation in Electronics. ACS Appl Mater Interfaces 2017.

24. Franz, R.; Wiedemann, G., Ueber die Wärme-Leitungsfähigkeit der Metalle. Annalen der Physik 1853, 165 (8), 497-531. 